\title{
Evaluation of the Cutaneous Lymphoma International Prognostic Index in patients with early stage mycosis fungoides*
}

\author{
Gustavo Moreira Amorim ${ }^{1,2}$, João Paulo Niemeyer Corbellini ${ }^{3}$, Danielle Carvalho Quintella ${ }^{4,5}$, \\ Tullia Cuzzi ${ }^{5,6}$, Márcia Ramos-e-Silva ${ }^{3,7}$
}

DOI: http:/ / dx.doi.org/10.1590/abd1806-4841.20187134

\begin{abstract}
BACKGROUND: Mycosis fungoides (MF) is the most common subtype of cutaneous T-cell lymphoma. TNMB system is the staging method used in MF, and it not only guides therapeutic management, but represents the main prognostic factor. In order to improve the prognostic evaluation, the Cutaneous Lymphoma International Prognostic Index (CLIPi) was proposed. Овлестіve: To evaluate the performance of CLIPi score for prognostic analysis in patients with early stage MF.

MeTHODs: This is a retrospective cross-sectional observational study, with exploratory analysis. The outcome variables were disease progression and related death.

RESULTS: One hundred and two patients were stratified according to CLIPi score, being the majority classified as low risk. Patients with intermediate or high risk presented disease progression more frequently than those with low risk (PR: $1.2 / \mathrm{p}=$ 0.004 / 95\% CI: 1.0 - 1.6). The same did not occur with the variable related death. In addition, survival rates were not consistent with risk stratification.

StUdy Limitations: Small sample and its retrospective analysis.

ConCLUSIONS: Since CLIPi score was proposed, four other studies that we could consult showed conflicting results, similar to the present study. Further studies are necessary for a recommendation of its use.
\end{abstract}

Keywords: Lymphoma; Lymphoma, T-cell, cutaneous; Mycosis fungoides

\section{INTRODUCTION}

Mycosis fungoides (MF) is the most common subtype of cutaneous T-cell lymphoma (CTCL) and has a behavior classified as indolent, with an overall survival in five years of $88 \% .^{1-4}$

The current staging is through the TNMB system, proposed by the Mycosis Fungoides Cooperative Group (MFCG) and subse- quently revised by the International Society for Cutaneous Lymphomas (ISCL) and by the European Organization of Research and Treatment of Cancer (EORTC) (Charts 1 and 2). In it, the letter $\mathrm{T}$ represents the cutaneous involvement and extent of the disease; $\mathrm{N}$, lymph node involvement; $\mathrm{M}$, the presence or absence of metastasis;

Received 23 March 2017.

Accepted 23 June 2017.

* Work conducted at the Dermatology and Anatomical Pathology Divisions, Hospital Universitário Clementino Fraga Filho, Universidade Federal do Rio de Janeiro, Rio de Janeiro (RJ), Brazil.

Financial support: None.

Conflict of interest: None.

1 Program of Post-graduation in Anatomical Pathology, Hospital Universitário Clementino Fraga Filho, Faculdade de Medicina, Universidade Federal do Rio de Janeiro, Rio de Janeiro (RJ), Brazil.

Outpatient clinic of Dermatology, Faculdade de Medicina, Universidade do Sul de Santa Catarina, Palhoça (SC), Brazil.

Dermatology Division, Hospital Universitário Clementino Fraga Filho, Universidade Federal do Rio de Janeiro, Rio de Janeiro (RJ), Brazil.

Pathology Division, Hospital Federal de Bonsucesso, Rio de Janeiro (RJ), Brazil.

Department of Pathology, Universidade Federal do Rio de Janeiro, Rio de Janeiro (RJ), Brazil.

Instituto Nacional de Infectologia Evandro Chagas, Fundação Oswaldo Cruz, Rio de Janeiro (RJ), Brazil.

Discipline of Dermatology, Faculdade de Medicina, Universidade Federal do Rio de Janeiro, Rio de Janeiro (RJ), Brazil.

MaILING AdDress:

Gustavo Moreira Amorim

E-mail: gustavomoreiraamorim@hotmail.com

(C)2018 by Anais Brasileiros de Dermatologia 


\section{CHART 1: TNMB staging of MF-type CTCL}

\section{Cutaneous involvement}

T1

T2

T3

T4

Lymph node involvement

No

N1

N2

N3

Nx

Visceral involvement

M0

M1

Peripheral blood involvement

B0

B1

B2
Patches, papules and/or plaques limited to $10 \%$ of the body surface area T1a (Patches)/T1b (Patches and plaques)

Patches, papules and/or plaques involving more than $10 \%$ of the body surface area T2a (Patches)/T2b (Patches and plaques)

One or more tumors (equal to or larger than $1 \mathrm{~cm}$ )

Confluence of erythema involving at least $80 \%$ of the body surface area

No abnormalities in the peripheral lymph nodes

Presence of abnormal lymph node. Dutch grade I on histopathology. N1a (negative clone)/ N1b (positive clone)

Presence of abnormal lymph node. Dutch grade II on histopathology. N2a (negative clone)/ $\mathrm{N} 2 \mathrm{~b}$ (positive clone)

Presence of abnormal lymph node. Dutch grade III or IV on histopathology. Negative or positive clone. Dutch histopathology grading system for the classification of lymph node involvement is recognized by ISCL/EORTC

Presence of abnormal lymph node, with no histological evaluation/confirmation

No visceral involvement

Presence of visceral involvement, confirmed by histopathology. The organ involved must be specified

Absence of peripheral blood involvement (equal to or less than $5 \%$ of atypical lymphocytes in the peripheral blood - Sézary cells). B0a (negative clone)/B0b (positive clone)

Presence of more than $5 \%$ of atypical lymphocytes, not fulfilling criteria for B2. B1a (negative clone)/B1b (positive clone)

Presence of 1,000 Sézary cells per cubic millimeter or more in the peripheral blood, with positive clone

Adapted: Olsen E et al., 2007.5

CHART 2: TNMB staging of MF-type CTCL

\begin{tabular}{|lcccc|}
\hline Staging & $\mathbf{T}$ & $\mathbf{N}$ & $\mathbf{M}$ & $\mathbf{B}$ \\
\hline IA & 1 & 0 & 0 & 0 or 1 \\
IB & 2 & 0 & 0 & 0 or 1 \\
IIA & 1 or 2 & 1 or 2 & 0 & 0 or 1 \\
IIB & 3 & 0 or 2 & 0 & 0 or 1 \\
III & 4 & 0 or 2 & 0 & 0 or 1 \\
IIIA & 4 & 0 or 2 & 0 & 0 \\
IIIB & 4 & 0 or 2 & 0 & 1 \\
IVA1 & 1 or 4 & 0 or 2 & 0 & 2 \\
IVA2 & 1 or 4 & 3 & 0 & 0 or 2 \\
IVB & 1 or 4 & 0 or 3 & 1 & 0 or 2 \\
\hline
\end{tabular}

Adapted: Olsen E et al., 2007. ${ }^{5}$

and B, extension to peripheral blood. ${ }^{4,5}$ TNMB staging system, albeit not ideal, remains as the main prognostic factor. ${ }^{5}$

At the time of diagnosis, most patients have early stage disease, defined by the TNMB staging as stage IA (T1N0M0), IB
(T2N0M0) or IIA (T1 or 2N1 or 2M0) (Charts 1 and 2). Four large published cohorts found in descending order, 78.3\%, 75.4\%, 71.5\% and $66 \%$ of patients with stage IA-IIA at the time of diagnosis. ${ }^{6-9}$

In prognostic terms, patients diagnosed with stage $1 \mathrm{~A} \mathrm{MF}$ have survival rates similar to those of the general population when

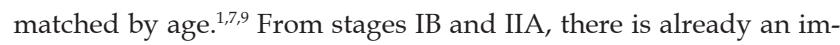
pact on the survival, even though they are still conceptually classified as early stage MF. A cohort study published by Desai et al. in 2015 with 393 patients, demonstrated a 5-year survival of $86.8 \%$ and $90.3 \%$ for stages IB and IIA, respectively. ${ }^{6}$ Similar results were found in the study published by Agar et al., with 1,502 patients, where 5 -year survival was $84 \%$ for IB and $78 \%$ for IIA. ${ }^{10}$ The drop in survival occurs more significantly from stage IIB, when MF are no longer considered early stage and start to be considered as having advanced MF. In the previously mentioned cohorts, 5-year survival in stage IIB was of $28.1 \%$ and $47 \%$, respectively. ${ }^{6,10}$

Approximately 20 to $25 \%$ of patients with stage I MF progressed to more advanced stages of the disease, with significant impairment of survival. Predicting which patients are under a higher risk of progression is still challenging, since there are no adequate prognostic markers. ${ }^{11}$ 
With the aim of improving the prognostic assessment of MF patients, Benton et al. suggested the implementation of a prognostic score, known as Cutaneous Lymphoma International Prognostic Index (CLIPi) (Chart 3). ${ }^{12}$ For this purpose, they studied 1,503 cases, published by Agar et al..$^{10}$ Validation of the score involved 1,221 outpatients. The score is differentiated for early stage and late stage MF an utilizes independent prognostic factors, with more statistical importance. They are: male gender and age, evaluated in both stages of MF; plaque lesions, folliculotropism, and N1 lymph node involvement (TNMB) for early stage MF, and B1/B2, N2/N3 visceral involvement for late stage MF. According to the presence of these characteristics, all scoring the same value (1 point), the patient is stratified into: low risk ( 0 to 1 , i.e., none or at least 1 factor present), intermediate risk (2, i.e., 2 factors present) and high risk ( 3 to 5 , i.e., between 3 and all 5 factors present). We highlight that the combination of the factors does not alter the proposed stratification, i.e., when 2 points are reached, either by being male and having plaque lesions, or by age and folliculotropism, it is still considered intermediate risk and so forth. ${ }^{12}$

The objective of this study was to evaluate the performance of CLIPi score as a prognostic factor in a sample of early stage MF patients (stages IA and IB only), treated at the Sector of Photodermatology, Dermatology Division, Hospital Universitário Clementino Fraga Filho - Universidade Federal do Rio de Janeiro (HUCFF/ UFRJ), and compare our data to the other studies published.

\section{METHODS}

This is an observational, cross-sectional, retrospective, exploratory data analysis study. The population of the study comprehended patients seen at the Sector of Photodermatology at HUCFF/ UFRJ, between January 2000 and December 2015, who were diagnosed with MF.

It was considered a case of MF the patient who had the three following criteria:

\begin{tabular}{|c|}
\hline Chart 3: CLIPi score \\
\hline Early stage MF: \\
\hline Male gender \\
\hline Age $>60$ years \\
\hline Plaques \\
\hline Folliculotropism \\
\hline N1/NX (TNMB staging) \\
\hline Interpretation: 0 - 1 (low risk) $/ 2$ (intermediate risk) $/ 3$ - 5 (high risk) \\
\hline Late stage MF: \\
\hline Male gender \\
\hline Age $>60$ years \\
\hline B1/B2 (TNMB staging) \\
\hline N1/NX (TNMB staging) \\
\hline Visceral involvement \\
\hline $\begin{array}{l}\text { Interpretation: } 0 \text { - } 1 \text { (low risk)/2 (intermediate risk)/3 - } 5 \text { (high risk) } \\
\text { Adapted: Benton EC et al., 2013. }{ }^{12}\end{array}$ \\
\hline
\end{tabular}

- Patches (only changes in the color of the skin, with no relief or texture changes) or plaques (raised, flat lesion, larger than $1 \mathrm{~cm}$ in diameter), that were hypopigmented or erythematous-coppery, with or without scaling, of different sizes and preferably affecting photoprotected areas; ${ }^{13}$

- Chronic course (at least 6 months), persistent or progressive;

Consistent histopathology (taking into consideration the clinical-pathological correlation). The presence of a lymphoid infiltrate along the dermal-epidermal junction, accompanied by epidermal invasion of lymphocytes without spongiosis or lymphocyte atypia (large, irregular, hyperchromatic nuclei) can be included in this situation.

Were excluded from the analysis patients with:

-Staging IIA or higher (i.e., disease not restricted to the skin); - Insufficient data in the patients file;

- Positive serology for HTLV 1/2;

- Diagnosis of other associated lymphomas;

- Follow-up of less than 5 years.

The dependent variables analyzed were:

- Progression of the disease as staging, classified as qualitative, dichotomous and nominal. Since stage IA and IB patients were included in the study, it was considered disease progression those who evolved to stage IIA onwards.

- Death related to the disease (being the lymphoma itself the cause of death, or deaths related to complications of systemic therapies used). The variable 682disease-related death was also treated in a qualitative, dichotomous and nominal fashion.

The independent variables studied were established by Benton et al. for the development of a specific early stage MF CLIPi score $(\text { Chart } 3)^{12}$ :

- Male gender;

- Age over 60 years;

- Plaque lesions;

- Folliculotropism.

All independent variables were treated in a qualitative, dichotomous and nominal fashion. Lymph node spread was not considered, since only TNMB IA or IB patients were included (Charts 1 and 2).

According to the number of independent variables present, the patients were classified in low, intermediate and high risk, as per the interpretation proposed by the authors of the article who support the prognostic score. Subsequently, low-risk patients were differentiated from those of intermediate and high risk, allowing for the creation of double entry tables and statistical analysis.

The data selected were grouped into printed spreadsheets and digitalized with Excel 2011 (Microsoft ${ }^{\circledR}$ Excel $^{\circledR}$ for Mac 2011/ Version: 14.2.0). Data were analyzed with the aid of the statistical software SPSS, version 24.0. the studies of the association between categorical data and dependent variables were performed using chi-squared test or Fischer's exact test. As measures of association, prevalence ratios and their respective confidence intervals (CI: 95\%) were calculated. The criterion of significance used was the $5 \%$ level.

The study is in accordance with the resolution 466/12 of the National Council of Health. It is registered at Plataforma Brasil and was approved by the Ethics Committee at HUCFF/UFRJ (CAAE 
59235916.9.0000.5257). The researches were responsible for the privacy and confidentiality of the data collected, fully preserving patient anonymity.

\section{RESULTS}

One hundred and two patients were included out of a total of 135 patients selected from a patient registry seen at the Sector of Photodermatology.

Among the records of 33 excluded patients, 17 had incomplete data or insufficient follow-up; 10 had other diagnoses (parapsoriasis, lymphomatoid papulosis, cutis laxa) and 6 had positive serology for HTLV 1/2.

Among the 102 patients studied, 30 (29.4\%) presented disease progression during follow-up and 8 died (7.8\%) from the disease or from treatment-related complications.

Only the presence of plaque lesions had a significant $p$-value regarding the association with stage progression in a bivariate analysis. However, confidence interval values included 1.0. This association was not maintained regarding the occurrence of MF-related deaths (Table 1).

As for the distribution of CLIPI score according to disease progression and disease-related deaths, the results are shown in table 2. Patients with intermediate and high risk evolved to disease progression in $35.9 \%$ and $40 \%$ of the cases, respectively. Lower rates of survival were seen in intermediate risk patients $(89.7 \%)$. The analysis in double entry table, differentiating the risks into associated low and intermediate and high risk and their correlation with the outcome variables is shown in table 3. The combined group of intermediate- and high-risk patients had a prevalence ratio of 1.4 $(p=0.04)$.

\section{DISCUSSION}

An observational, cross-sectional study was performed in order to evaluate the CLIPi score as a prognostic factor in patients with the diagnosis of early stage MF, seen at the Sector of Photodermatology at HUCFF/UFRJ.

The sample was made of 102 stage IA or IB MF patients, undergoing follow-up for at least 5 years. The majority was classified as low-risk according to the CLIPi score.

The main prognostic factor continues to be the staging according TNMB classification. ${ }^{5,14}$ In this sample, only the presence of plaque lesions was associated to a higher frequency of stage progression. In a study being prepared for publication conducted by the same authors of this study, using the same cases, besides the presence of plaque lesions, disease involvement of more than $10 \%$ of the body surface area, abnormal lactic dehydrogenase and beta-2-microglobulin, besides stage IB itself, were identified as poor prognosis factors. Supporting these findings, advanced age at diagnosis, male gender, intense pruritus, lymph node enlargement, peripheral eosinophilia, the presence and the size of Pautrier's microabscesses, folliculotropism, large cells suggestive of transformation on histology, immunohistochemistry evidencing loss of positivity of CD7 and CD5 in mature T-cells, positivity for CD4 and positivity for CD30 higher than $15 \%$ and, finally, detection of TCR clonality in peripheral blood were described as factors of worse prognosis. $13,3,6,9,10,11,14,15$

As previously described, to improve prognostic assessment Benton et al. suggested the prognostic score CLIPi. From their initial findings and validation in a cohort with a significant number of patients, the authors identified that, regarding early stage MF, there was a statistically significant difference in terms of disease progression and survival, when the patients were divided into three groups. Those classified as low-risk had a 10-year survival of $90.3 \%$,

TABLE 1: Analysis of the frequency of independent variables and calculation of the prevalence ratio with the association to dependent variables

\begin{tabular}{|c|c|c|c|c|c|c|c|c|c|c|c|c|}
\hline \multirow{3}{*}{$\begin{array}{l}\text { Variables } \\
\text { independent }\end{array}$} & & & \multicolumn{10}{|c|}{ Dependent variables } \\
\hline & \multicolumn{6}{|c|}{ Progression } & \multicolumn{5}{|c|}{ Related death } & \multirow[b]{2}{*}{$95 \%$ CI } \\
\hline & $\mathrm{N}^{\mathrm{o}}$ & $\begin{array}{c}\text { Freq } \\
(\%)\end{array}$ & $\mathrm{N}^{\mathrm{o}}$ & $\begin{array}{c}\text { Freq } \\
(\%)\end{array}$ & P R & $P$ & CI 95\% & $\mathrm{N}^{\circ}$ & $\begin{array}{c}\text { Freq } \\
(\%)\end{array}$ & PR & $P$ & \\
\hline Male & 55 & 53.9 & 19 & 34.5 & 1.4 & 0.1 & $0.7-2.7$ & 4 & 7.3 & 0.8 & 0.5 & $0.2-3.2$ \\
\hline$>60$ years & 50 & 49.0 & 16 & 32.0 & 1.1 & 0.3 & $0.6-2.1$ & 3 & 6.0 & 0.6 & 0.3 & $0.1-2.4$ \\
\hline Plaques & 56 & 54.9 & 22 & 39.3 & 1.3 & 0.01 & $1.061-1.745$ & 6 & 10.7 & 1.0 & 0.2 & $0.9-1.1$ \\
\hline Folliculotropism & 6 & 5.9 & 1 & 16.7 & 0.5 & 0.4 & $0.1-3.3$ & 0 & 0 & - & - & - \\
\hline $\mathrm{N} 1 / \mathrm{Nx}$ & 0 & 0 & - & - & - & - & - & - & - & - & - & - \\
\hline
\end{tabular}

N: number. Freq: frequency, expressed in percentage. PR: prevalence ratio. 95\% CI: 95\% confidence interval

TABLE 2: Distribution of the frequency of classification of the CLIPi score according to the frequencies of progression and related death

\begin{tabular}{|c|c|c|c|c|c|c|c|c|c|}
\hline \multirow[b]{2}{*}{ CLIPi score: } & \multirow[b]{2}{*}{$\mathbf{N}$} & \multirow[b]{2}{*}{ Freq $(\%)$} & \multicolumn{3}{|c|}{ Progression } & \multicolumn{3}{|c|}{ Related death } & \multirow{2}{*}{$\begin{array}{l}\text { 5-year survival } \\
(\%)\end{array}$} \\
\hline & & & $\mathbf{N}$ & Freq $(\%)$ & $\mathbf{P}$ & $\mathbf{N}$ & Freq $(\%)$ & $\mathbf{P}$ & \\
\hline 1. Low risk & 43 & 42.2 & 8 & 18.6 & 0.1 & 3 & 7 & 0.7 & 93 \\
\hline 2. Intermediate risk & 39 & 38.2 & 14 & 35.9 & & 4 & 10.3 & & 89.7 \\
\hline 3. High risk & 20 & 19.6 & 8 & 40 & & 1 & 5 & & 95 \\
\hline
\end{tabular}

$\mathrm{N}$ : Number. Freq: frequency, expressed in percentage 


\begin{tabular}{|c|c|c|c|c|c|c|c|c|c|c|}
\hline \multirow[b]{2}{*}{ CLIPi score } & \multicolumn{5}{|c|}{ Progression } & \multicolumn{5}{|c|}{ Related death } \\
\hline & $\mathbf{N}^{0}$ & Freq $(\%)$ & PR & $P$ & $95 \% \mathrm{CI}$ & $\mathbf{N}^{0}$ & Freq $(\%)$ & PR & $P$ & $95 \% \mathrm{CI}$ \\
\hline 1. Low risk & 8 & 18.6 & 0.4 & 0.04 & $0.2-1.0$ & 3 & 7 & 0.8 & 0.7 & $0.2-3.2$ \\
\hline 2. Interm. and high & 22 & 37.3 & 1.2 & & $1.018-1.655$ & 5 & 8.6 & 1.0 & & $0.9-1.1$ \\
\hline
\end{tabular}

Interm. and high: Group combining intermediate and high risk. N: number. Freq: frequency, expressed in percentage. PR: prevalence ratio. $95 \%$ CI: $95 \%$ confidence interval

and $84.5 \%$ did not show stage progression during that time. Those with intermediate risk had a 10 -year survival of $76.2 \%$, and $68.8 \%$ did not progress. Finally, high-risk patients had a 10 -year survival of $48.9 \%$, and $54.5 \%$ of progression-free survival. The CLIPi score as a prognostic tool was able to refine the evaluation and, therefore, was recommended by the authors, with the reservation that it should be tested in multicentric studies. ${ }^{12}$

This study found $42.2 \%$ low-risk, $38.2 \%$ intermediate-risk, and $19.6 \%$, therefore minority, high-risk patients in a sample of 102 patients, according to the CLIPi score. When low, intermediate and high risk were compared to disease progression and subsequently to disease-related death, even though there was a higher percentage of progressing cases among those classified as high-risk, this difference was not statistically significant $(p=0.1)$. In the same way, there was no difference between the groups regarding mortality. Paradoxically, the highest percentage of 5-year survival was among high-risk patients. Therefore, in our sample, this datum conflicted with what was proposed in the study by Benton et al. ${ }^{12}$

When the intermediate- and high-risk cases were grouped and compared to low-risk cases, a higher prevalence of stage progression was identified in the higher risk group, with an estimated prevalence ratio of 1.2-fold, and this difference was statistically significant $(p=0.04)$. however, the confidence interval does not allow for substantiation of this hypothesis since it contains the value 1.0. Thus, in the present study there was a tendency of a higher prevalence of progression in patients with early stage MF who did not have a low risk CLIPi score. The same was not seen for the occurrence of death.

In 2015, Wernham et al. published the findings of a study with 86 early stage MF patients. The patients were divided into two groups: non-progression and progression of the stage (to at least IIB). CLIPi score was tested in a group with 60 patients, of which 30 had progressed. The 30 remaining patients, classified as non-progressing, were matched by age with the progression group. A higher percentage of low-risk patients was identified among those who did not progress, however, this difference was not statistically significant. ${ }^{11}$

Three other studies that analyzed the CLIPi score were published in 2016. Sanz-Bueno et al. studied the performance of the CLIPi prognostic tool in a cohort with 82 early stage MF patients. The 10 -year survival found was of $86 \%$ for low-risk patients, $91 \%$ for intermediate-risk and $64 \%$ for high-risk. Similar to what we found in our study, there was also a paradoxical finding with those cases in that among with those with intermediate risk, there was a higher survival when compared to those with low risk. Progression-free survival in 10 years was of $77 \%, 74 \%$ and $69 \%$, respectively. The differences, however, were not significant. ${ }^{16}$
Danish et al. published their results of the application of CLIPi in a cohort with 390 patients. Of those, 305 had early stage MF. Five-year survival was $98.4 \%, 88.2 \%$ and $60.8 \%$ for low-risk, intermediate-risk and high-risk patients, respectively. In the same way, disease-free survival after 5 years was $99.0 \%, 88.1 \%$ and $65.8 \%$, respectively. In both outcomes, the difference between the groups was statistically significant $(p<0.0001)$. The authors considered that the score had a good performance in terms of risk stratification for early stage MF patients, and as Benton et al., suggested its validation in appropriate prospective studies. ${ }^{17}$

Finally, Nikolaou et al. published their findings regarding the study of prognostic factors in a series of 393 patients with early stage MF. All 393 were classified according to the CLIPi score. The authors identified that, after 5 years, according to CLIPi, high-risk patients had a 4.19-fold risk of stage progression and 5.25-fold higher mortality risk, when compared to those with low risk, findings that showed a statistically significant p-value and adequate confidence interval. However, it was not possible to demonstrate a difference for the group with intermediate risk in comparison to that with low risk. ${ }^{14}$

We point out that it is crucial to advance in the prognostic evaluation of MF patients, since a small but significant percentage, even if classified as early stage, courses with disease progression, affecting survival.

\section{CONCLUSION}

We conclude that the results of the usage of the CLIPi score in our sample were conflicting, as demonstrated by the relevant literature, therefore requiring new studies for the definition of its applicability. It is important to highlight that the limitations of this study are its retrospective design and its relative small sample. It was mentioned in the studies consulted the initiative of a prospective, multicentric study in Europe, with the goal of analyzing the performance of PROCLIPi; apparently an adjustment to improve accuracy of CLIPi score. We suggest waiting for those publications. $\square$

\section{AKNOWLEDGEMENTS}

We would like to thank Prof. Dr. Nurimar Conceição Fernandes by the thorough review. 


\section{REFERENCES}

1. Wilcox RA. Cutaneous T-cell lymphoma: 2014 update on diagnosis, riskstratification, and management. Am J Hematol. 2014;89:837-51.

2. Yamashita T, Abbade LP, Marques ME, Marques SA. Mycosis fungoides and Sézary syndrome: clinical, histopathological and immunohistochemical review and update. An Bras Dermatol. 2012;87:817-28.

3. Jawed SI, Myskowski PL, Horwitz S, Moskowitz A, Querfeld C. Primary cutaneous T-cell lymphoma (mycosis fungoides and Sézary syndrome): part II. Prognosis, management, and future directions. J Am Acad Dermatol. 2014;70:223:e1-17.

4. Willemze R, Jaffe ES, Burg G, Cerroni L, Berti E, Swerdlow SH, et al. WHO-EORTC classification for cutaneous lymphomas. Blood. 2005;105:3768-85.

5. Olsen E, Vonderheid E, Pimpinelli N, Willemze R, Kim Y, Knobler R, et al. Revisions to the staging and classification of mycosis fungoides and Sezary syndrome: a proposal of the International Society for Cutaneous Lymphomas (ISCL) and the cutaneous lymphoma task force of the European Organization of Research and Treatment of Cancer (EORTC). Blood. 2007;110:1713-22.

6. Desai M, Liu S, Parker S. Clinical characteristics, prognostic factors, and survival of 393 patients with mycosis fungoides and Sézary syndrome in the southeastern United States: a single-institution cohort. J Am Acad Dermatol. 2015;72:276-85

7. van Doorn R, Van Haselen CW, van Voorst Vader PC, Geerts ML, Heule F, de Rie M, et al. Mycosis fungoides: disease evolution and prognosis of 309 Dutch patients. Arch Dermatol. 2000:136:504-10.

8. Talpur R, Singh L, Daulat S, Liu P, Seyfer S, Trynosky T, et al. Long-term outcomes of 1.263 patients with mycosis fungoides and Sézary syndrome from 1982 to 2009. Clin Cancer Res. 2012;18:5051-60.

9. Kim YH, Liu HL, Mraz-Gernhard S, Varghese A, Hoppe RT. Long-term outcome of 525 patients with mycosis fungoides and Sezary syndrome: clinical prognostic factors and risk for disease progression. Arch dermatol. 2003;139:857-66.
10. Agar NS, Wedgeworth E, Crichton S, Mitchell TJ, Cox M, Ferreira S, et al. Survival outcomes and prognostic factors in mycosis fungoides/Sézary syndrome: validation of the revised International Society for Cutaneous Lymphomas/ European Organization for Research and Treatment of Cancer staging proposal. $J$ Clin Oncol. 2010;28:4730-9.

11. Wernham AG, Shah F, Amel-Kashipaz R, Cobbold M, Scarisbrick J. Stage I mycosis fungoides: frequent association with a favourable prognosis but disease progression and disease specific mortality may occur. Br J Dermatol. 2015;173:1295-7.

12. Benton EC, Crichton S, Talpur R, Agar NS, Fields PA, Wedgeworth E, et al. A cutaneous lymphoma international prognostic index (CLIPi) for mycosis fungoides and Sezary syndrome. Eur J Cancer. 2013;49:2859-68.

13. Cardili RN, Roselino AM. Elementary lesions in dermatological semiology: literature review. An Bras Dermatol. 2016:91:629-33.

14. Nikolaou V, Papadavid E, Patsatsi A, Siakantaris M, Economidi A, Marinos L, et al. Prognostic indicators for mycosis fungoides in a Greek population. $\mathrm{Br} \mathrm{J}$ Dermatol. 2017;176:1321-30.

15. Scarisbrick JJ, Kim YH, Whittaker SJ, Wood GS, Vermeer MH, Prince HM, et al. Prognostic factors, prognostic índices and staging in mycosis fungoides and Sézary syndrome: where are we now? Br J Dermatol. 2014;170:1226-36.

16. Sanz-Bueno J, Lora D, Monsálvez V, Maroñas-Jiménez L, Postigo C, RodríguezPeralto JL, et al. The new Cutaneous Lymphoma International Prognostic index (CLIPi) for early mycosis fungoides failed to identify prognostic groups in a cohort of Spanish patients. Br J Dermatol. 2016;175:794-6.

17. Danish HH, Liu S, Jhaveri J, Flowers CR, Lechowicz MJ, Esiashvili N, et al. Validation of cutaneous lymphoma international prognostic index (CLIPi) for mycosis fungoides and Sézary syndrome. Leuk Lymphoma. 2016;57:2813-9.

\section{AUTHORS'CONTRIBUTIONS}

Gustavo Moreira Amorim

iD ORCID 0000-0001-6067-9463

Critical review of the literature; Design and planning of the study; Collecting, analysis and interpretation of data; Statistical analysis; Preparation and writing of the manuscript João Paulo Niemeyer Corbellini (iD) ORCID 0000-0001-8104-3915

Critical review of the literature; Intellectual participation in propaedeutic and/or therapeutic conduct of studied cases; Statistical analysis

Danielle Carvalho Quintella

iD ORCID 0000-0001-9013-9417

Critical review of the literature; Intellectual participation in propaedeutic and/or therapeutic conduct of studied cases; Critical review of the manuscript

\footnotetext{
Tullia Cuzzi ORCID 0000-0002-3331-5290

Effective participation in research orientation; Design and planning of the study; Critical review of the manuscript; Approval of the final version of the manuscript Márcia Ramos-e-Silva $\quad$ (iD) ORCID 0000-0003-1625-0760 Effective participation in research orientation; Design and planning of the study; Critical review of the manuscript; Approval of the final version of the manuscript
}

How to cite this article: Amorim GM, Niemeyer-Corbellini JP, Quintella DC, Cuzzi T, Ramos-e-Silva M. Evaluation of the Cutaneous Lymphoma International Prognostic Index in patients with early stage mycosis fungoides. An Bras Dermatol. 2018;93(5):680-5. 